\title{
Report on the Round Table Conference on Medical Care Management of Patients with Neurocutaneous Syndromes
}

On 9 November 2004 the Round Table Conference on Medical Care Management of Patients with Neurocutaneous Syndromes was held in Warsaw. The Conference was organised by the Polish Neurocutaneous Syndromes Clinics Initiating Group and the Children's Memorial Health Institute in Warsaw. The heads of the departments involved in the treatment of patients with phakomatoses and delegates of the Polish Ministry of Health and the Polish National Health Fund participated in the Meeting. Members of patients' families, gathered in three paternal organizations: Neurofibromatosis 1, Tuberous Sclerosis and Sturge-Weber Syndrome have also taken the floor. Altogether, approximately 120 persons attended the Conference.

Currently, the Polish Neurocutaneous Syndromes Clinics Initiating Group consists of representatives of 3 centres in Poland (Warsaw, Bydgoszcz and Szczecin), which assembled the major number of patients with neurocutaneous syndromes within the last two decades. In the future, it is planned to extend the Group to include other centres involved in neurocutaneous syndromes in Poland.

In the first part of the Conference, Professor Sergiusz Jóźwiak, Professor Mariusz Wysocki and Professor Jan Lubiński presented diagnostic and therapeutic standards in three most frequent neurocutaneous syndromes: tuberous sclerosis, neurofibromatosis and von Hippel-Lindau disease. They all emphasised that the knowledge about these syndromes in medical milieu is still insufficient and that regular control examinations in phakomatoses are required to diagnose neoplasms at their earliest stage.

In the second part of the Conference, methods to improve medical care of neurocutaneous syndrome patients were discussed. Both family members and specialists stressed that specialised clinical centres resembling Phakomatoses Clinics existing in some countries are needed to establish comprehensive management of the patients. The problems with performance of control examinations (including CT and MRI scans), which are often expensive, were also raised. The lack of respective regulations of payment by the National Health Fund was pointed out among the reasons of insufficient number of these examinations.
The possibility of creating national registers of selected phakomatoses, which could help to assess the exact frequency of these diseases in Poland, was also discussed. In many countries, genetic examinations are performed with respect to neurocutaneous syndromes; whereas in Poland, except for von Hippel-Lindau disease, such examinations are not available. This issue was discussed as well. Delegates of the National Health Fund declared that it would be possible to obtain individual promises of reimbursement of costs of such examinations performed abroad.

The Conference was the first public discussion on the management of patients with neurocutaneous syndromes in Poland. 